\title{
Physically-Coherent Extraction of Mitral Valve Chordae
}

\author{
Daryna Panicheva ${ }^{1}$, Pierre-Frederic Villard ${ }^{1,2}$, Peter E Hammer ${ }^{3}$, Marie-Odile Berger ${ }^{1}$ \\ ${ }^{1}$ Université de Lorraine, CNRS, Inria, LORIA, Nancy, France \\ ${ }^{2}$ Harvard School of Engineering and Applied Sciences, Cambridge, MA, USA \\ ${ }^{3}$ Harvard Medical School, Boston, MA, USA
}

\begin{abstract}
Surgical repair of the mitral valve is challenging as it is difficult to anticipate the outcome of any modification of the valve structure, particularly the tendinous chordae. Recent works on computer-based models of mitral valve behavior rely on manual extraction of the complex valve geometry, which is tedious and requires a high level of expertise. On the contrary, we propose a method to segment the chordae with little human supervision. The effectiveness of our approach is shown by comparing our segmentation to the manual delineation via the simulation of the closed valve state.
\end{abstract}

\section{Introduction}

The mitral valve (MV) malfunction is one of the frequent causes for the development of cardiovascular disease. The MV is a heart valve situated between the left atrium and the left ventricle assuring one-directional blood flow during the cardiac cycle. It is composed of two asymmetrical leaflets supported by the system of tethering chordae attached to the papillary muscles on the ventricle wall (see figure 1).

The treatment of MV-related diseases often implies surgical repairs. Due to the anatomical complexity of the valve, clinicians have poor ability to predict the intervention outcome. Computer modeling of the valve behavior could then be helpful as a part of preoperative planning.

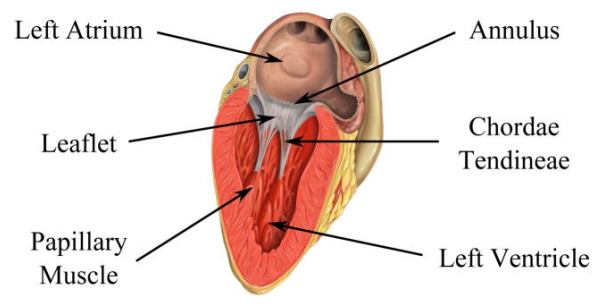

Figure 1. Mitral valve anatomy.

The MV modeling includes valve geometry representa- tion, definition of the valve mechanical properties and setting the boundary conditions for the model. In this work we are going to focus on the geometry extraction step.

First studies on the mitral valve modeling were based on simplified geometries used to represent its complex anatomy ([1]). With the development of the image acquisition modalities, various groups focus on subject-specific models where the valve components are extracted from the image data. In recent works ([2], [3], [4], [5]) the precise segmentation of the valve anatomy together with complex material models allows to obtain promising simulations. However, the segmentation is still performed in a manual or semi-manual way remaining a laborious task. Taking into account the high intra-subject variance and the complexity of the valve anatomy, the segmentation results can significantly vary depending on the expert.

Therefore, an automatic solution for the MV components extraction is a relevant problem. In this work we propose a method for semi-automatic segmentation of the MV chordae aiming to minimize the human supervision in the process (section 2.1). The leaflets are extracted manually. The close MV state is simulated with a finite-element method (FEM) model (section 2.2). The chordae segmentation is validated by comparing the simulation results to those obtained with manually segmented chordae (section 3). As data we used micro-CT scan of a porcine heart. The image was acquired ex-vivo in the closed valve state simulated with air flow trough the aorta (pressure $=120 \mathrm{~mm}$ $\mathrm{Hg})$.

\section{Methods}

\subsection{Valve components segmentation}

The structures in the data can be divided into three groups: flat structures (leaflets and ventricle walls), tubular structures (chordae) and bifurcations (zones of chordae branching). Chordae and their bifurcations are the only tubular structures in the image, i.e. having more than one connected component (CC) of the boundaries (in red in figure 2.B). Thereby, in order to semi-automatically ex- 

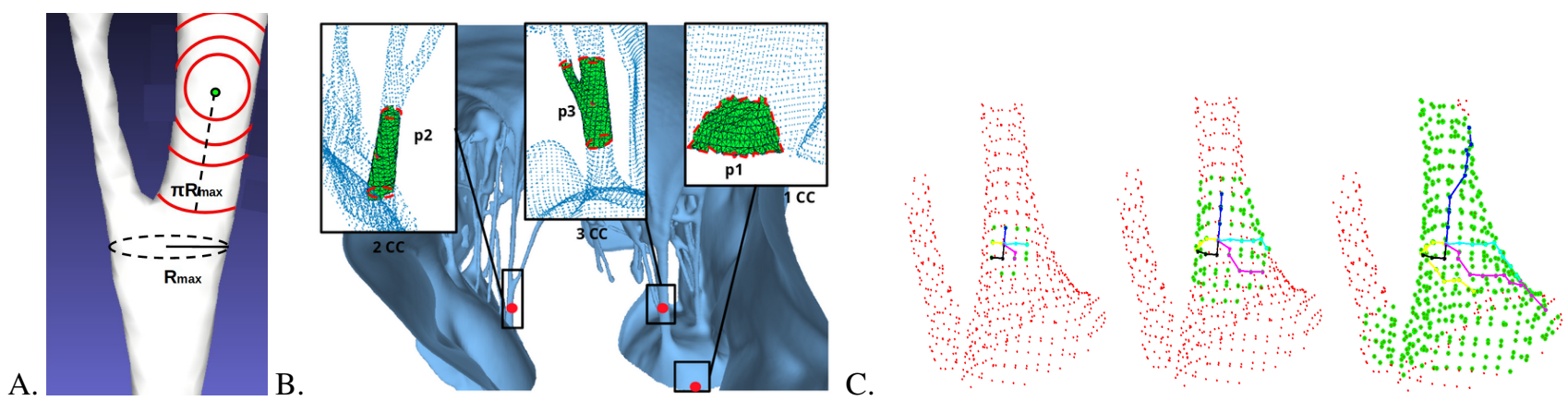

Figure 2. A. Submesh definition: center point in green, lines uniting points being on the same geodesic distance in red. B. Labeling process: points to be labeled in red, corresponding submeshes in green. C. Submesh extraction step by in-mesh propagation procedure: line segments - geodesic path approximations, extracted submesh in green.

tract the chordae we use an approach based on topological properties as described in [6].

At the first stage the initial micro-CT scan is denoised and filtered with the Canny edge filter [7]. After that, a mesh is obtained with the Screened Poisson method ([8]).

Next, points of the mesh are labeled according to the number of $\mathrm{CC}$ in the boundaries of the corresponding submeshes. The submesh is centered on the point to be labeled and limited by the geodesic distance bounded by the halfcircumference of the largest tubular structure in the data. The latter is approximated by $\pi R_{\max }, R_{\max }$ being the maximum radius of the chordae (see figure 2.A). Thereby, $p_{1}$ in figure 2.B would be labeled as non-chordae (one boundary CC), $p_{2}$ - as chordae (two boundary $\mathrm{CC}$ ) and $p_{3}$ as bifurcation ( 3 boundary $\mathrm{CC}$ ).

Defining the points being at the same geodesic distance (iso-geodesic contour) is very time consuming in practice for large datasets (above $300 \mathrm{k}$ points in our case). In the sake of segmentation speed enhancement, it was substituted with a in-mesh propagation approach. Specifically, for a point to be labeled its direct neighborhood is extracted giving the directions for geodesic paths approximations (lines connecting the center point with the isogeodesic contour). At the following steps, closest points by euclidean distance are added to the paths until the sum of segments length reaches the threshold given by $\pi R_{\max }$. In figure 2.C the initial (left), intermediate (center) and final (right) steps of the procedure are given, green points correspond to the resulting submesh.

Since the submeshes being centred on the neighboring points overlap, all the points in the submesh are labeled and not only the center one. The points used as centers of submeshes are uniformly distributed in the volume. This does not completely exclude submeshes superposition but reduces its number considerably, therefore decreases computational time.

The segmentation result obtained with the method is shown in figure 3. Chordae and their bifurcations are in red and non-chordae structures - in blue. The extracted chordae contain few false positives and can be used for the further geometry representation in the biomechanical model. Specifically, with the use of a RANSAC-based approach not described in this work the chordae are represented as a set of connected cylinders with bifurcation zones. The center-lines of the cylinders and the centers of gravity of the bifurcation zones are used then for the simulation (in blue in figure 4).

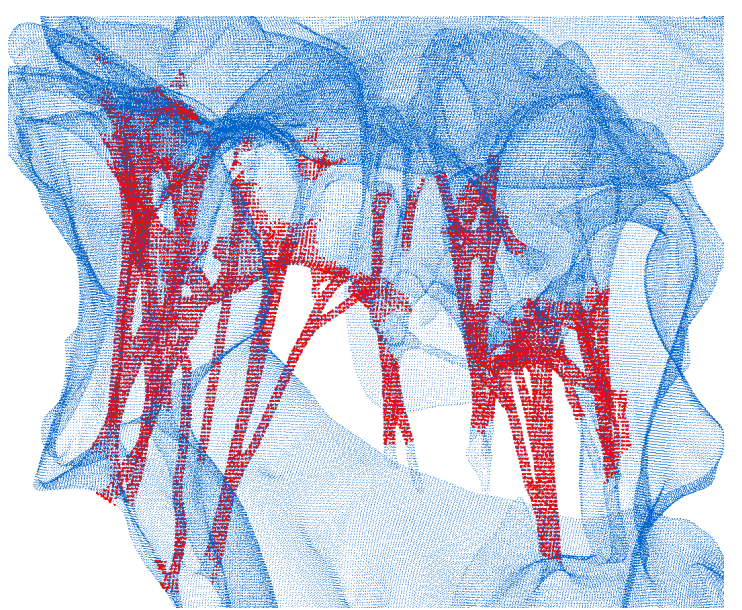

Figure 3. Chordae segmentation results: chordae in red, non-chordae in blue.

The valve leaflets were segmented manually with ITKSnap software [9] followed by the extraction of the exterior surface of the valve. Final steps included smoothing and decimation of the resulting leaflets mesh (the triangulated mesh in figure 4). The annulus points (hinge line of the leaflets) were defined manually (in yellow in figure 4).

Due to the presence of ambiguous transition zones between chordae and leaflets, a gap occur. In order to connect the chordae to the leaflet a fan-like attachment was performed (the black segments in figure 4) similarly to the approach proposed in [3]. 


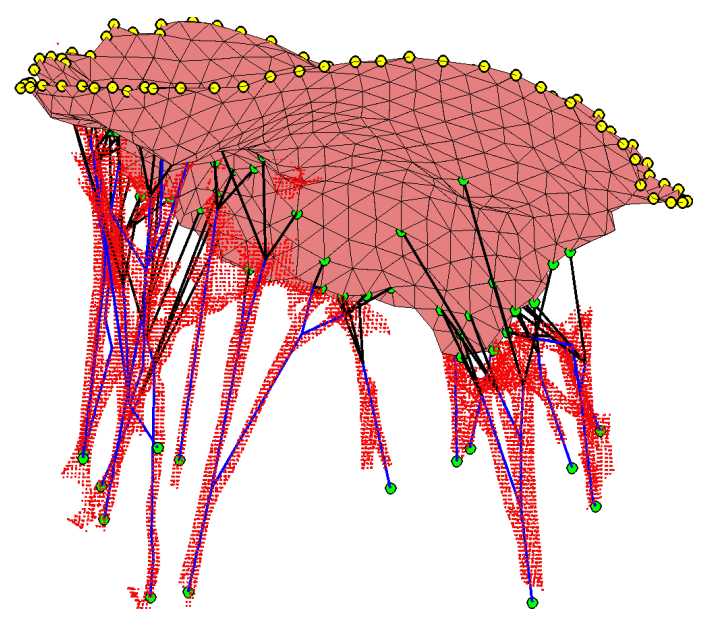

Figure 4. MV geometry representation in the biomechanical model: leaflets as a triangulated mesh, chordae as line segments.

\subsection{Biomechanical model}

The valve behavior was simulated with a biomechanical model based on the framework proposed in [10] except that the mass-spring system has been replaced by a FEM continuum system similarly to [11]. It is a structural model (with no fluid-structure interaction). Physiological behavior is simulated by mechanical forces. The following forces are computed for each triangle vertex:

- pressure forces resulting from the pressure applied to the leaflet during peak systole (closure). It corresponds to a pseudo-static state. It can be computed for each vertex as the weighted sum of the pressure applied to the faces connected to this vertex. The direction is normal to the surface.

- internal forces which are obtained with the FEM technique. First, the strain tensor is computed with the vertices displacement using the Green tensor. Secondly, the stress is computed from the strain with a constitutive law using the 2nd Piola-Kirchhoff stress tensor. The parameters are the same as in [11]. The forces are eventually computed from the stress using the relationship defined in [12].

- tension forces which are applied to the leaflet vertex tethered to the chordae. The chordae are modeled as an elastic material with a high Young's modulus.

- contact forces which are used to avoid the collisions along the zone of leaflet coaptation. The collision detection is based on computing bounding boxes for all the triangles and detecting if they are overlapping. Contact force is computed by an exponential contact force prior to penetration and a linear penalty force after penetration [10].

The leaflet vertices along the valve annulus are fixed. The sum of forces is used to compute the acceleration according to Newton's second law. The new positions are computed by solving the system of partial differential equations using semi-implicit numerical integration method [13].

\section{Results}

We propose the following criteria classically used by the community for the validation. Firstly, it is necessary to verify that the simulated behavior is coherent. This means that the valve should stay sealed when all the chordae are extracted and included in the model. Similarly, the leaflets could bulge and the structure could leak in the case of chordae ruptures (chordae set not complete). Secondly, chordae manually delineated from the initial microCT scan were used as ground truth. Thus the quality of the semi-automatic segmentation is assessed by comparison of the simulation results to those where the MV geometry was obtained by manual segmentation. The simulated cases include the valve with the entire chordal set (healthy valve) and partially removed chordae (pathological case), with the semi-automatic and manual segmentation, respectively (see figure 5.A). The quantitative criteria used are the mechanical stress distribution (von Mises criteria) and the bulging volume, i.e. the volume above the annulus plane, similarly to [14]. The annulus plane corresponds to a surface fitted to the annulus points with a thin-plate smoothing spline (in gray in figure 5.B).

The results show that the simulations with manual and semi-automatic chordae extraction lead to similar final valve configurations. With the whole chordae set the valve stays sealed (top raw on the figure 5.C). In the case of chordae deletion, leaks and bulging appear (bottom raw on the figure 5.C). The bulging increases by 2.5 and 2.1 times for the manual and automatic segmentation, respectively (see figure 5.C). The von Mises stress distribution for both cases is as well comparable: the error in mean value between manual and automatic methods is $3.3 \%$ (all chordae) and $4.2 \%$ (partly absent chordae) (see figure 5.C).

\section{Conclusion and discussion}

A method allowing the semi-automatic mitral valve chordae segmentation was proposed. The only parameter required corresponds to the size of the largest chord in the image. The segmentation was validated trough out the simulation with a FEM-based model. It was shown that the simulation outcomes obtained with the valve geometry being segmented semi-automatically are comparable to the results in the case of completely manual pipeline. It gives reason to believe in the potential application of the semi-automatic approaches in practice.

Future work will concern the development of a semiautomatic solution for leaflet segmentation, which will allow us to validate the proposed approach on more datasets. 

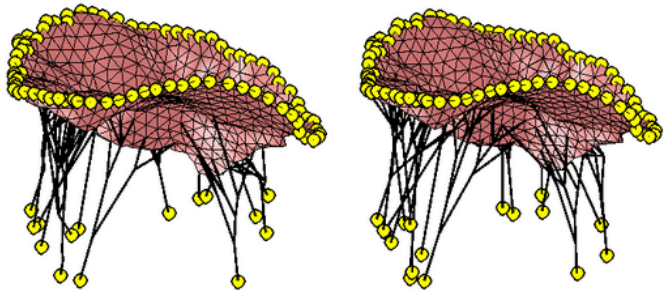

A.
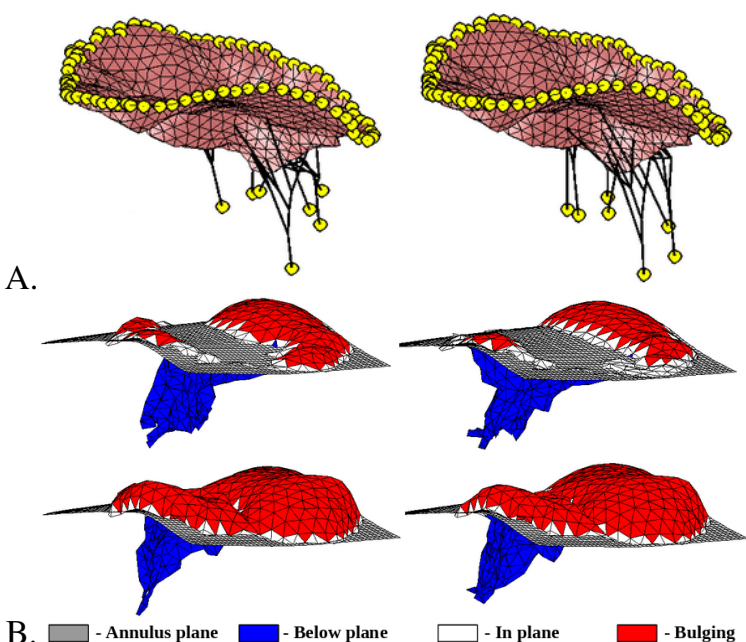

B.

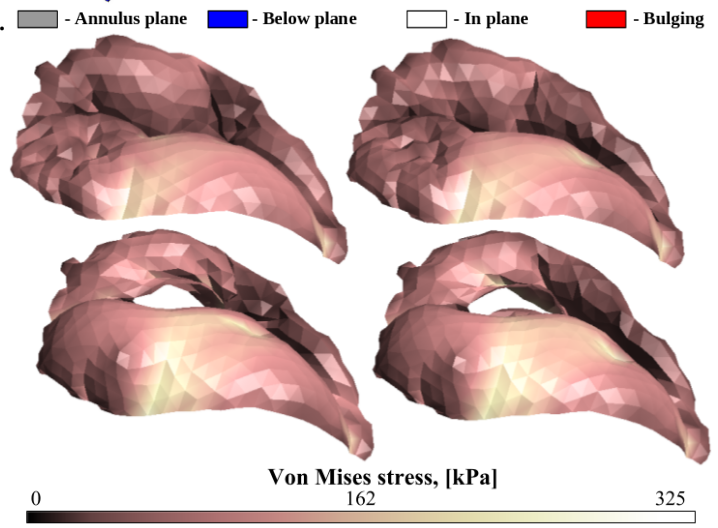

Figure 5. Comparison of the simulation outcomes for semi-automatic (left) and manual (right) segmentations with all (top) and ruptured (bottom) chordae: A. Simulation cases. B. Bulging volumes. C. Von Mises stress distribution.

\section{Acknowledgments}

This work was performed in part at the Harvard University Center for Nanoscale Systems (CNS), a member of the National Nanotechnology Coordinated Infrastructure Network (NNCI), which is supported by the National Science Foundation under NSF ECCS award no. 1541959.

\section{References}

[1] Kunzelman K, Cochran R, Chuong C, Ring W, Verrier E, Eberhart R. Finite element analysis of the mitral valve. The journal of heart valve disease 1993;2(3):326 - 340.

[2] Toma M, Einstein DR, Bloodworth IV CH, Cochran RP, Yoganathan AP, Kunzelman KS. Fluid structure interaction and structural analyses using a comprehensive mitral valve model with $3 \mathrm{~d}$ chordal structure. International Journal for Numerical Methods in Biomedical Engineering 2017;33(4).

[3] Drach A, Khalighi A, Sacks M. A comprehensive pipeline for multiresolution modeling of the mitral valve: Validation, computational efficiency, and predictive capability. International Journal for Numerical Methods in Biomedical Engineering 2017;34.

[4] Feng L, Qi N, Gao H, Sun W, Vazquez M, Griffith BE, Luo $\mathrm{X}$. On the chordae structure and dynamic behaviour of the mitral valve. IMA Journal of Applied Mathematics 2018; 83(6):1066-1091.

[5] Villard PF, Hammer PE, Perrin DP, Del Nido PJ, Howe R. Fast image-based mitral valve simulation from individualized geometry. The International Journal of Medical Robotics and Computer Assisted Surgery 2018;14(2).

[6] Panicheva D, Villard PF, Berger MO. Toward an automatic segmentation of mitral valve chordae. In SPIE Medical Imaging, volume 10953. 2019; 4p.

[7] Canny J. A computational approach to edge detection. IEEE Trans Pattern Anal Mach Intell June 1986;8(6):679_ 698.

[8] Kazhdan M, Hoppe H. Screened poisson surface reconstruction. ACM Trans Graph July 2013;32(3):29:1-29:13.

[9] Yushkevich PA, Piven J, Cody Hazlett H, Gimpel Smith R, Ho S, Gee JC, Gerig G. User-guided 3D active contour segmentation of anatomical structures: Significantly improved efficiency and reliability. Neuroimage 2006;31(3).

[10] Hammer PE, del Nido PJ, Howe RD. Anisotropic massspring method accurately simulates mitral valve closure from image-based models. In Functional Imaging and Modeling of the Heart. Berlin, Heidelberg: Springer Berlin Heidelberg, 2011; 233-240.

[11] Hammer PE, Chen PC, del Nido PJ, Howe RD. Computational model of aortic valve surgical repair using grafted pericardium. Journal of Biomechanics 2012;45(7).

[12] Taylor RL, Oñate E, Ubach PA. Finite Element Analysis of Membrane Structures. Textile Composites and Inflatable Structures 2005;47-68.

[13] Press WH, Teukolsky SA, Vetterling WT, Flannery BP. Numerical Recipes 3rd Edition: The Art of Scientific Computing. 3 edition. Cambridge University Press, 2007.

[14] Jolley MA, Hammer PE, Ghelani SJ, Adar A, Sleeper LA, Lacro RV, Marx GR, Nathan M, Harrild DM. Threedimensional mitral valve morphology in children and young adults with marfan syndrome. Journal of the American Society of Echocardiography 2018;31(11):1168 - 1177.

Address for correspondence:

Daryna Panicheva

615 Rue du Jardin-Botanique, 54506 Vandoeuvre-les-Nancy, France

daryna.panicheva@loria.fr 